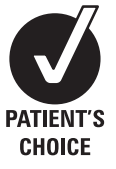

${ }^{1}$ Department of Rehabilitation Medicine, University of Melbourne, Royal Melbourne Hospital, Melbourne, Victoria Australia; ${ }^{2}$ School of Rural Health, University of Melbourne, Melbourne, Victoria, Australia; ${ }^{3}$ Department of Clinical Epidemiology and Health Service Evaluation Unit, Royal Melbourne Hospital, Melbourne, Victoria, Australia; ${ }^{4}$ Centre for Neuroscience, The University of Melbourne, Parkville, Victoria, Australia

Correspondence to:

Dr Fary Khan, Department of Rehabilitation Medicine, University of Melbourne, Royal Melbourne Hospital, Poplar Road Parkville, Melbourne, VIC 3052, Australia; fary.khan@mh.org.au

Received 30 August 2007 Revised 16 April 2008 Accepted 5 May 2008 Published Online First 5 June 2008

\title{
Effectiveness of rehabilitation intervention in persons with multiple sclerosis: a randomised controlled trial
}

\author{
F Khan, ${ }^{1}$ J F Pallant, ${ }^{2}$ C Brand, ${ }^{3}$ T J Kilpatrick ${ }^{4}$
}

\author{
ABSTRACT \\ Objective: A stratified, randomised, waitlist controlled \\ study over 12 months assessed the effectiveness of \\ rehabilitation in persons with multiple sclerosis (MS) in an \\ Australian community cohort.
}

Methods: Patients with definite MS $(n=101)$ recruited from a tertiary hospital database, randomised to a treatment group $(n=49)$ for individualised rehabilitation programme or a control waitlist group $(n=52)$.

Functional Independence Measure (FIM) was used to assess "activity" while the Multiple Sclerosis Impact Scale (MSIS-29) and General Health Questionnaire (GHO28) assessed "participation" and quality of life (OoL). Assessments were at baseline and 12 months.

Results: Analysis of data from 98 patients (treatment $n=48$, control $n=50$ ) showed reduced disability in the treatment group, with statistically significant differences in post-treatment FIM motor scores for the two groups $(p<0.001)$. There was a clinical and statistically significant improvement in FIM (motor) total scores $(p<0.001)$, and the FIM motor domains of: transfer $(p<0.001)$, locomotion $(p<0.001)$, self-care $(p<0.001)$ and the FIM cognitive subscale $(p<0.016)$. In the treated group, $70.8 \%$ improved compared with $13 \%$ of controls. Significantly more patients in the control group deteriorated over the study period $(58.7 \%$ vs $16.7 \% ; p<0.001)$. There were no differences between the control and treatment group scores on the MSIS-physical $(p=0.18)$, MSIS-psychological $(p=0.45)$ or GHO subscales.

Conclusion: An individualised rehabilitation programme reduces disability in persons with MS compared with no intervention. The impact of rehabilitation on $0 \mathrm{~L}$ needs further evaluation. More information on the effectiveness of the various components of the multidisciplinary rehabilitation programmes are now needed.

Australian clinical trials registry:

Trials registration number: ACTRN012605000676617.

Multiple sclerosis (MS) is a chronic demyelinating disease of the central nervous system and a common cause of neurological disability, affecting 2.5 million persons worldwide. ${ }^{1}$ It has an unpredictable course and is usually progressive in nature. About $50 \%$ of persons require a gait aid and $10 \%$ a wheelchair within 15 years of onset, ${ }^{2}$ and $90 \%$ will have significant functional limitation 25 years after onset. ${ }^{3}$

Persons with MS (pwMS) are expected to have a normal lifespan and live for many decades with a range of problems. The World Health Organization developed the International Classification of Functioning, Disability and Health (ICF) ${ }^{4}$ which defines a common language for describing the impact of disease at different levels: impairment, limitation in activity and participation. Within this framework, MS related impairments (weakness, spasticity) can limit "activity" or function (decreased mobility, incontinence) and "participation" (work, family, social reintegration). These have a cumulative effect over time and cause considerable distress to the pwMS and their families, reducing quality of life $(\mathrm{OoL})$. There are significant socioeconomic implications with increased demand for health care, social and vocational services, and caregiver burden.

Despite major advances in MS care and disease modifying therapies, no longer term benefit on disability and participation have yet been demonstrated. The supportive and symptomatic management provided by multidisciplinary (MD) rehabilitation programmes remain the mainstay of treatment. A recent systematic review ${ }^{5}$ provided support for $\mathrm{MD}$ rehabilitation programmes for pwMS using outcomes based on the ICF. The eight randomised (RCT) and clinical controlled trials ${ }^{6-14}$ in this review highlighted a number of methodological difficulties with RCTs in rehabilitation settings. These relate to heterogeneous MS patient populations, difficulty with quantifying interventions, patient attrition and lack of control group, variability with follow-up and assessment, and lack of appropriate and sensitive outcome measures. Although seven (of eight) studies used randomisation procedures, concealed allocation of treatment and blinded outcome assessors were reported in only one study. ${ }^{90}$ Furthermore, blinding of patients and care providers in all studies was inadequate. Storr et $a l^{15}$ used a double blind design to assess the short term efficacy of $\mathrm{MD}$ inpatient rehabilitation compared with a control group and reported no significant difference. This study was underpowered, and confounding factors included variation in indication for treatment and in the reliability and responsiveness of outcome measures used.

In the current study we developed methodology for a stratified, randomised, waitlist controlled study over 12 months using blinded care providers and outcome assessors to compare the effectiveness of $\mathrm{MD}$ rehabilitation in pwMS in an Australian community cohort.

\section{METHODS}

\section{Participants and setting}

The study was conducted at the Royal Melbourne Hospital (RMH), a tertiary referral centre in Victoria, Australia, and approved by its research committee. The RMH MS programme provides acute neurological and intensive MD rehabilitation treatment for inpatients and ambulatory care 5 days per week for $3-6$ weeks. The aim is to 
improve patient symptoms, maximise function and address factors relating to $\mathrm{QoL}$ and participation.

Patients were recruited from the RMH MS database; participant selection and methodology have been described previously. ${ }^{16}$ Independent neurologists confirmed MS (McDonald criteria), ${ }^{17}$ MS stage and severity using the Expanded Disability Status Scale (EDSS). ${ }^{18}$ These participants were mobile in the community, with EDSS scores for mobility between 2 and 8, and cognition based on the Kurtzke Functional Systems (KFS range 0-2). Participants with cognition scores of KFS $>2$, those with relapse in the 3 months prior to recruitment and rehabilitation in the 6 months before admission were excluded.

\section{Procedure}

\section{Randomisation}

A total of 204 patients were eligible for this study and randomised to control and treatment groups using a computer generated sequence, stratified by EDSS scores. All patients were invited (by mail) to participate in the project. A total of 101 people replied and returned signed consent forms. All patients were then mailed an MS information package. They were informed that it could take between 6 and 12 months before they received rehabilitation. The treatment group $(n=49)$ received the individualised inpatient (IP) or outpatient $(\mathrm{OP})$ rehabilitation programme while the waitlist patients were the control group $(n=52)$.

\section{Assessment interviews}

Patients were assessed in their homes using a structured format by three independent trained researchers (one physician, two research officers) over a 6-week period (January-February 2005). They were not in contact with the acute neurology or the rehabilitation treating team. They did not share information about participants or assessments, and received independent clinical record forms at each interview. They completed demographic, functional and QoL assessments using standardised instruments (see measures). These assessments were in a randomised order to minimise systematic bias. Wherever possible patients were assessed at the same time of day (before noon). These interviews took approximately $1.5 \mathrm{~h}$, with appropriate rest breaks. The assessors did not prompt patients but provided assistance for those who had difficulty with completing the questionnaires.

The control group was reassessed 12 months later while the treatment group were evaluated at 12 months after completion of their rehabilitation programme. The assessors had no access to previous assessments or to patient treatment schedules. Patients were instructed to make no comments on whatever treatment they received and to only report any relapse or concurrent illness.

\section{Treatment schedules}

Patients in the treatment group received comprehensive $\mathrm{MD}$ rehabilitation over a 12 month period. The treating therapy team assessed each patient's potential to benefit from either an IP or OP programme, based on clinical features, individual need and accessibility to services. The treating therapy team was not aware of patients in the trial and assessed these patients along with the usual referrals from the community. Those in the control group were not unduly disadvantaged, as patients referred for rehabilitation from the community with no acute need for rehabilitation usually wait between 6 and 8 months for a stay in hospital. Those in the control group who needed acute rehabilitation were offered treatment.

The $\mathrm{MD}$ programme included intensive treatment beyond symptomatic management of $\mathrm{MS}$, and aimed to educate patients (and caregivers), and improve "activity" and "participation" within the limits of disease. ${ }^{19}$ This programme included individual, achievable, functional goal oriented $\mathrm{MD}$ treatment with active involvement of the patient and family. The interventions offered were wide ranging (education, health promotion, bladder retraining and mobilisation).

The 5 day IP rehabilitation programme included 3 h of therapy per day, involving relevant disciplines based on patient need and team consensus. Compliance with the IP programme was defined as patient attendance in $>90 \%$ of treatment sessions, comprising two blocks of 45 min sessions with physiotherapy and occupational therapy daily. Other sessions included half hour sessions with speech pathology, neuropsychology and social work 3 times/week or more (as required) for between 3 and 6 weeks. The individualised OP programme offered a lower intensity of therapy. An a priori compliance with OP treatment was defined as patient attendance in $>80 \%$ of treatment sessions which included half hour blocks of therapy sessions (physiotherapy, occupational therapy, social work and speech pathology), 2-3 times per week as needed for up to 6 weeks. Subsequently they were involved in maintenance programmes (stretching, home exercises) similar to those undertaken by the control group. The methods used in therapy were individually tailored to meet patient needs (physiotherapy included muscle stretching, balance and gait training, while occupational therapy treatments involved fatigue management and functional retraining in tasks of daily living).

Adverse effects of rehabilitation were recorded (falls or injury during treatment). A dedicated phone number was made available to all participants during office hours to address questions or concerns, and the relevant specialist was contacted if required. The control group received an 8 weekly monitoring phone call for information about medical and hospital visits in the previous month.

\section{Measurement}

The ICF ${ }^{5}$ was used as a conceptual basis for choice of best outcomes for measurement. Activity was assessed with the Functional Independence Measure (FIM), ${ }^{20}$ while the Multiple Sclerosis Impact Scale (MSIS-29) ${ }^{21}$ and General Health Questionnaire (GHO-28) ${ }^{22}$ assessed participation and QoL.

FIM $^{20}$ assesses function and need for assistance in 18 items in motor and cognitive domains. Each item is rated on a scale of 1 to 7 ( 1 =total assistance, $5=$ supervision, $7=$ independent). The score reflects burden of care in each area measured. The FIM scores were based on actual performance of a task on a daily basis, rather than each individual optimum performance.

MSIS-2921 measures the physical and psychological impact of MS from the patient's perspective. Total scores for both subscales are generated by summing individual items (scored 1-5) with high scores indicating greater impact.

GHQ-28 $8^{22}$ requires respondents to compare their current health to their "usual state" using a four point response scale for each item within its four subscales (somatic symptoms, anxiety, social dysfunction, severe depression). Higher scores indicate greater symptomatology.

\section{Statistical analysis}

The primary outcome was the impact of the rehabilitation programme on disability assessed by the FIM motor subscale. 
Table 1 Baseline patient characteristics for the treatment and control groups, and for those who did and did not receive treatment

\begin{tabular}{|c|c|c|c|c|}
\hline Variable & $\begin{array}{l}\text { Randomised to } \\
\text { treatment group } \\
(\mathrm{n}=49)\end{array}$ & $\begin{array}{l}\text { Randomised to } \\
\text { control group } \\
(n=52)\end{array}$ & $\begin{array}{l}\text { Received } \\
\text { treatment } \\
(\mathrm{n}=61)\end{array}$ & $\begin{array}{l}\text { Did not receive } \\
\text { treatment } \\
(\mathrm{n}=40)\end{array}$ \\
\hline n (\% female) & $31(63.3)$ & $41(78.8)$ & $40(65.6)$ & $32(80)$ \\
\hline Age (y) (mean (SD) (range)) & $49.5(8.64)(30-63)$ & $51.1(9.66)(29-65)$ & $49.7(8.96)(30-65)$ & $51.2(9.51)(29-63)$ \\
\hline \multicolumn{5}{|l|}{ Disease (n (\%)) } \\
\hline Relapsing-remitting & $13(26.5)$ & $18(34.6)$ & $17(27.9)$ & $14(35)$ \\
\hline Secondary progressive & $29(59.2)$ & $27(51.9)$ & $36(59)$ & $20(50)$ \\
\hline Primary progressive & $7(14.3)$ & $7(13.5)$ & $8(13.1)$ & $6(15)$ \\
\hline Time since diagnosis (y) (mean (SD)) & $10.69(6.33)$ & $9.73(7.99)$ & $10.52(6.61)$ & $9.7(8.11)$ \\
\hline No of relapses in past 2 y (mean (SD)) & $1.07(1.18)$ & $1.83(2.15)$ & $1.17(1.17)$ & $1.89(2.21)$ \\
\hline \multicolumn{5}{|l|}{ EDSS (n (\%)) } \\
\hline $0-3$ & $7(14.3)$ & $12(23.1)$ & $8(13.1)$ & $11(27.5)$ \\
\hline $3.5-6.0$ & $27(55.1)$ & $32(61.5)$ & $36(59)$ & $23(57.5)$ \\
\hline $6.5+$ & $15(30.6)$ & $8(15.4)$ & $17(27.9)$ & $6(15)$ \\
\hline FIM motor (mean (SD)) & $74.67(13.43)$ & $78.5(12.14)$ & $75.2(12.66)$ & $78.87(13.04)$ \\
\hline FIM cognitive (mean (SD)) & $32.35(2.76)$ & $33.5(2.19)$ & $32.56(2.68)$ & $33.55(2.21)$ \\
\hline MSIS physical (mean (SD)) & $50.31(18.02)$ & $48.89(19.43)$ & $51.45(17.21)$ & $46.46(20.76)$ \\
\hline MSIS psychological (mean (SD)) & $19.27(8.43)$ & $18.81(7.32)$ & $19.92(8.26)$ & $17.54(7.0)$ \\
\hline GHO anxiety (mean (SD)) & $5.86(3.79)$ & $5.54(4.09)$ & $6.46(4.18)$ & $4.53(3.24)$ \\
\hline GHO depression (mean (SD)) & $3.98(4.54)$ & $2.98(4.17)$ & $4.03(4.57)$ & $2.6(3.9)$ \\
\hline GHO somatic (mean (SD)) & $6.96(3.69)$ & $6.63(3.94)$ & 7.07 (3.57) & $6.38(4.15)$ \\
\hline GHO social (mean (SD)) & $8.73(2.82)$ & $8.02(2.5)$ & $8.69(2.8)$ & $7.88(2.41)$ \\
\hline
\end{tabular}

EDSS, Expanded Disability Status Scale; FIM, Functional Independence Measure; GHQ, General Health Questionnaire; MSIS

Multiple Sclerosis Impact Scale.

For an $80 \%$ chance to detect a five point difference in FIM from baseline to 12 months in the intervention compared with the control group, assuming that SD for change is 8.5 and is similar in both groups, 46 patients in each group were needed for recruitment. The estimate was based on a two sided $\alpha=0.05$.

Additional analyses were also conducted on the subscale scores for FIM (motor, cognitive) and MSIS. Treatment and control groups were compared, with patients assigned according to their initial randomisation, irrespective of their subsequent compliance to the protocol.

Complete information was not available for all patients at the second data collection point. MS can have a fluctuating nature and patients could be expected to deteriorate over the 12 month study period. Assigning the last observation carried forward ${ }^{23}$ values to missing data would therefore bias results by assuming a favourable outcome (no deterioration) for missing cases. A "complete case" approach was used, with patients providing information at both time points included in each of the analyses.

The primary analyses were conducted using analysis of covariance (ANCOVA), comparing the post-treatment scores for the control and treatment groups, with the baseline score as a covariate..$^{24}$ Secondary analyses were conducted on the individual FIM motor subscales using multivariate analysis of covariance (MANCOVA), to assess the impact of the programme across a set of related outcome measures.

FIM scores were negatively skewed and were subjected to log transformation and reflection. Bonferroni adjustments were made to the alpha level used to assess statistical significance. ${ }^{25}$

A series of independent group $t$ tests were used to compare change scores (baseline minus post treatment) for the control and treatment groups for the FIM, MSIS and GHO. Effect size statistics (Cohen's d) ${ }^{26}$ were determined by subtracting the mean change score for the two groups and dividing by the pooled baseline SD. ${ }^{27}$ The $\chi^{2}$ statistic compared percentages of cases in the control and intervention groups who improved, remained the same or deteriorated.
During the course of the study a number of patients in the control group $(n=12,23 \%)$ required treatment. In addition to the per protocol analyses described earlier, additional analyses were conducted to compare patients who received treatment with those who did not.

\section{RESULTS}

Of 204 eligible patients there was no difference in age, gender or EDSS scores between those who consented and those who did not.

\section{Baseline characteristics}

The sample of 101 patients included 52 randomised to the control and 49 to the treatment group. One patient in the treatment group withdrew consent after the first visit, while two patients in the control group were lost to follow-up (one died, one withdrew consent). Data from 98 patients were available for statistical analysis (treatment $n=48$, control $\mathrm{n}=50$ ). Patient characteristics and comparison of baseline scores for both groups are summarised in table 1. The two groups were well matched for all variables. The treatment group included more severely affected persons (EDSS $>6.5$ ) who are expected to respond less well to treatment.

There was no difference in the follow-up intervals between the control (12 months) and treatment (12.8 months) groups. Only one patient in the control group had received rehabilitation in the previous 14 months.

\section{Rehabilitation programme}

Of the 49 patients in the treatment group, 24 received an individualised IP or OP $(n=25)$ programme. Mean length of the rehabilitation programme was 34 days (range 6-64). There was $96 \%$ and $89 \%$ compliance with treatment with the IP and OP programme, respectively, as per the a priori "compliance" definition. Additional speech pathology, neuropsychology and fatigue sessions were received by $60 \%$ of those who received the 
Table 2 Change scores for treatment and control groups for activity and participation

\begin{tabular}{|c|c|c|c|c|c|c|c|c|}
\hline \multirow[b]{2}{*}{ Scale } & \multicolumn{2}{|c|}{ Treatment } & \multicolumn{2}{|c|}{ Control } & \multirow{2}{*}{$\begin{array}{l}\text { Mean } \\
\text { difference }\end{array}$} & \multirow{2}{*}{$\begin{array}{l}95 \% \mathrm{Cl} \text { for } \\
\text { mean difference }\end{array}$} & \multirow[b]{2}{*}{ p Value } & \multirow{2}{*}{$\begin{array}{l}\text { Effect } \\
\text { size }\end{array}$} \\
\hline & $n$ & Mean (SD) & $n$ & Mean (SD) & & & & \\
\hline FIM motor & 48 & $-3.0(4.23)$ & 46 & $1.78(4.25)$ & 4.78 & 3.04 to 6.52 & $<0.001$ & 1.13 \\
\hline Transfers & 48 & $-0.75(1.28)$ & 48 & $0.50(1.13)$ & 1.25 & 0.76 to 1.74 & $<0.001$ & 1.04 \\
\hline Locomotion & 48 & $-0.63(1.28)$ & 47 & $0.21(1.16)$ & 0.25 & 0.34 to 1.34 & 0.001 & 0.69 \\
\hline Sphincter & 48 & $-0.33(.91)$ & 47 & $0.02(1.28)$ & 0.36 & -0.10 to .81 & 0.12 & 0.32 \\
\hline Self-care & 48 & $-1.29(2.05)$ & 47 & $0.92(2.56)$ & 2.21 & 1.27 to 3.15 & $<0.001$ & 0.95 \\
\hline FIM cognitive & 48 & $-0.15(1.46)$ & 48 & $0.42(1.09)$ & 0.563 & 0.04 to 1.08 & 0.035 & 0.44 \\
\hline \multicolumn{9}{|l|}{ MSIS } \\
\hline Psychological & 46 & $0.11(7.7)$ & 46 & $-1.09(6.5)$ & -1.20 & -4.16 to 1.77 & 0.43 & 0.44 \\
\hline Physical & 46 & $-1.13(16.9)$ & 46 & $2.30(12.68)$ & 3.44 & -2.76 to 9.63 & 0.27 & 0.23 \\
\hline \multicolumn{9}{|l|}{$\mathrm{GHO}$} \\
\hline Anxiety & 47 & $0.26(4.83)$ & 50 & $0.20(4.04)$ & -0.055 & -1.85 to 1.74 & 0.95 & 0.01 \\
\hline Depression & 47 & $0.13(4.42)$ & 50 & $0.34(3.09)$ & 0.212 & -1.32 to 1.74 & 0.78 & 0.06 \\
\hline Somatic & 46 & $1.15(4.48)$ & 50 & $-0.10(5.28)$ & -1.25 & -3.30 to .79 & 0.23 & 0.26 \\
\hline Social & 47 & $1.02(3.75)$ & 50 & $0.34(3.73)$ & -0.68 & -2.91 to .83 & 0.37 & 0.18 \\
\hline
\end{tabular}

Change scores were calculated by subtracting time 2 from time 1, therefore improvement is indicated by negative changes scores on the FIM and positive change scores on the MSIS.

The standardised treatment effect calculated as $\mathrm{D}=(\mathrm{mi}-\mathrm{mc}) / \mathrm{SD}$ pooled, where $\mathrm{mi}$ is the mean change score of the intervention group and $\mathrm{mc}$ is the mean change score of the control group.

FIM, Functional Independence Measure; GHQ, General Health Questionnaire; MSIS Multiple Sclerosis Impact Scale.

OP programme. In the treated group, all patients had a combined $1 \mathrm{~h}$ session with their "significant other" and treating team. There were no adverse events reported in either group.

\section{Outcome measurements for activity and participation}

A one way ANCOVA, comparing the post-treatment FIM motor scores for the control and treatment groups (with the baseline score as a covariate), was statistically significant ( $F$ (1, $91)=10.95, \mathrm{p}<0.001)$. A subsequent MANCOVA assessing each of the individual FIM motor subscales showed significant differences between the control and intervention groups $(p<0.01)$ for all aspects assessed (transfers, locomotion, sphincter control, self-care). A significant result was also obtained from ANCOVA analysis on the FIM cognitive scale $(p<0.016)$ but not for the MSIS physical $(p=0.18)$, MSIS psychological $(p=0.45$ ) or any of the GHO subscales.

\section{Outcome measurement change scores}

Change scores for FIM, MSIS and GHQ were calculated (time 1 minus time 2) for both groups (table 2). Compared with controls, the treated group showed statistically significant improvement in FIM total scores, and in the FIM domains of transfers, locomotion and self-care. Although the difference for the FIM cognitive scale was significant at $p<0.05$, it was not significant at the Bonferroni adjusted value of $0.005(0.05 / 11)$.

The percentage of patients who improved, showed no change and/or deteriorated in the FIM scores were calculated for each group. As expected, more patients in the treatment group improved compared with controls $(70.8 \%, 34 / 48$ vs $13 \%, 6 / 46)$, while more patients in the control group deteriorated over the study period $(58.7 \%, 27 / 46$ vs $16.7 \%, 8 / 48)$. This difference in proportions between the treatment and control groups was statistically significant $\left(\chi^{2}=32.5, \mathrm{df}=2, \mathrm{p}<0.001\right)$.

Twelve patients randomised to the control group required treatment. There was no difference between the patients in the control group that did compared with those who did not receive treatment in terms of gender, age, EDSS grouping, stage of the disease, years since diagnosis or mean scores on admission for the FIM subscales. There was, however, a significant difference in the GHO-anxiety $(p=0.001), G H Q$-total scores $(p=0.03)$ and in scores on the MSIS-29 psychological subscale $(p=0.04)$, with the group requiring treatment showing higher levels of distress at admission.

The primary ANCOVA analyses reported earlier comparing the control $(n=52)$ and treatment $(n=49)$ groups were repeated, comparing those patients that received treatment $(n=61)$ and those that did not receive treatment $(n=40)$. Consistent results were obtained, with significant differences between the groups being detected for the FIM motor $(p=0.001)$, the FIM domains of self-care $(p=0.001)$, sphincter $(p=0.01)$, transfers $(p=0.003)$, locomotion $(p=0.007)$, but not for the MSIS psychological ( $p=0.39)$, MSIS physical $(p=0.47)$ or $\mathrm{GHQ}$ subscales.

\section{DISCUSSION}

This study supports the effectiveness of an individualised rehabilitation programme for pwMS compared with no intervention. The treatment group showed reduction in disability and demonstrated important clinical differences in patient outcomes, with large treatment effect sizes for a number of FIM domains. This reduction in disability is consistent with previous reports, ${ }^{6-14} 2829$ but not all. ${ }^{15}$ Patients in the treated group improved in function, while those in the control group deteriorated during the study period. In contrast with a previous report, ${ }^{30}$ positive effects on emotional well being and QoL were not demonstrated at 12 months.

In our study, the treatment effect for the FIM mobility subscale was larger than expected, as pwMS are not usually expected to show this degree of improvement in mobility. There are a number of possible reasons for this. Compared with our study, patients in a previous study ${ }^{6}$ had a shorter average duration of rehabilitation (20 days), more severely disabled patients (median EDSS 6.5 (range 5-9)) and shorter follow-up time ( 6 weeks). Our study included a longer follow-up of community dwelling pwMS with adequate cognition (KFS 0-2), who could fully participate and learn compensatory strategies in the rehabilitation programme for maximum benefit. In addition, their next of kin also attended therapy. The longer duration and more intensive therapy, and caregiver involvement, may be important factors for improved outcomes. 
A recent double blind $\mathrm{RCT}^{15}$ over 6 weeks did not support the efficacy of $\mathrm{MD}$ rehabilitation. There was unequal patient distribution in the two groups, a high patient attrition rate and a more disabled control group, creating bias. Patients were not assessed prior to admission, so the indication for rehabilitation was not always clear. In addition, patients had higher EDSS scores (median 6.5 (range 0-8)) and received less therapy time (45 min daily) compared with our study and other reports. ${ }^{69}$ In our study, the patient attrition rate was low, both groups had comparable baseline characteristics and adequate cognition. Less severe cognitive impairment in pwMS has been associated with improved goal setting and rehabilitation outcomes. ${ }^{31} 32$ The responsiveness of our patient population to rehabilitation suggests the current triage process is effective in selecting those most likely to benefit from this resource intensive intervention. The additional benefit of incidental therapy (data not shown) is difficult to determine but the rates in both groups were comparable to those reported by Storr and colleagues. ${ }^{15}$ The significant difference in rates of deterioration between the intervention and control groups over 12 months supports the work of Freeman and colleagues. ${ }^{6}$ In addition to functional improvements, additional attention and environmental modification for the treatment group may have been a contributing factor to these differences.

Despite recent reports ${ }^{33}$ the MSIS-29 did not show changes in emotional well being and QoL. This may be due to "response shift phenomenon" 34 where the pwMS may reassess their perceived limitations of daily living and reset goals and consider the impact of their MS less marked than they thought previously.33 These "response shifts" can occur because of differences among people or changes within people regarding internal standards, values or conceptualisation of QoL.

The GHO is generic and may fail to discriminate important domains of QoL for pwMS. We previously reported under utilisation of health services, especially rehabilitation, unmet needs and a lower $\mathrm{QoL}$ in pwMS compared with healthy Australian subjects. ${ }^{16}$ Other studies report a lower QoL for pwMS with a longer disease duration, ${ }^{35} 36$ with secondary progressive $\mathrm{MS}^{37}$ and those with cognitive dysfunction. ${ }^{38}$ Over $50 \%$ of patients in this study had secondary progressive MS and had adequate cognition. The relatively long period before reassessment may have missed earlier positive effects on $\mathrm{QoL}$ that were not sustained. Furthermore, important changes related to community reintegration may not have been adequately detected. Comparison of rehabilitation outcomes for "participation" may now be possible with recent developments in ICF MS Core set. ${ }^{39}$ This comprises ICF categories that are relevant to most pwMS and can guide MD assessments, record the effect of intervention and allow comparison with other studies.

Meaningful comparisons with previous studies are limited by methodological issues, including differences in patient populations, study designs, the "black box" of complex intervention and different outcome measures. The outcomes achieved were at one centre in Victoria, Australia, and further studies are needed to confirm their generalisability. Although the changes in FIM scores for both groups were small, they were clinically relevant. The FIM scores of individuals with disability correlate highly with hours of care required. ${ }^{40}$ This was beyond the scope of the current study.

The issues of design in such $\mathrm{MD}$ health service interventions are complex. The "real life" clinical setting in this instance had its own set of challenges (operational issues relating to slot availability within the health service, appropriate staffing levels for timely intervention). Waitlist control groups have been criticised as patients know they are waiting and may be "disgruntled" with this treatment. However, as our patients were unaware of the "fast tracked" treated group, this would have mitigated this potentially negative effect.

To reduce a major source of bias, the treating therapists and assessors were blinded. The treating therapists provided treatment to patients but were unaware of the study. The assessors had no links with the rehabilitation or acute hospital team, and had no contact with one another or access to the initial scores. The time interval between the two assessments was adequate (12 months) to reduce the likelihood of spontaneous recovery or short term gains following the rehabilitation programme. While a comprehensive approach to outcome measurement was undertaken, we may have missed other important outcomes, such as impact on carers and families.

In conclusion, this stratified, randomised waitlist controlled study over 12 months supports individualised MD rehabilitation programmes in reducing disability in pwMS compared with no intervention. The impact of response shift phenomenon on QoL needs further exploration. More information about defining and characterising the components of "the black box" of clinical practice in rehabilitation now needed using multicentre trials.

Acknowledgements: We are grateful to the patients with MS and their families who participated in this study. We would like to thank T McPhail, K Vezzoso, M Tanner (randomisation, concealed allocation, recruitment and consent), L Spooner, C Paros, L Oskari (for patient interviews and assessments), T McPhail and Jason Pallant (data entry), L McGregor (for statistical advice) and the rehabilitation treating team RPC RMH, Professor L Turner Stokes and T Hale for advice in planning this study and W Briggs for assistance with preparation of this manuscript.

\section{Competing interests: None.}

Ethics approval: The study was approved by the research committee of the Royal Melbourne Hospital, Victoria, Australia.

\section{REFERENCES}

1. World Health Organization. Atlas: Country Resources for Neurological Disorders. Results of a collaborative study of World Health Organization and World Federation of Neurology. Programme for Neurological Diseases and Neurosciences Department of Mental Health and Substance Abuse. Geneva: World Health Organization, 2004.

2. Weinshenker BG, Bass B, Rice GP, et al. The natural history of multiple sclerosis: a geographically based study 2. Predictive value of early clinical course. Brain 1989:112:1419-28.

3. Frohman E, Racke M, van den Noort S. To treat or not to treat: the therapeutic dilemma of idiopathic minisymptomatic demyelinating syndromes. Arch Neurol 2000;57:1-6.

4. World Health Organization. International Classification of Functioning, Disability and Health: ICF. Geneva: World Health Organization, 2001.

5. Khan F, Turner Stokes L, Ng L, et al. Multidisciplinary rehabilitation for adults with multiple sclerosis (Review). Cochrane Database Syst Rev 2007;2:CD006036.

6. Freeman JA, Langdon DW, Hobart JC, et al. The impact of inpatient rehabilitation on progressive multiple sclerosis. Ann Neurol 1997;42:236-44.

7. Craig J, Young CA, Ennis $\mathrm{M}$, et al. A randomised controlled trial comparing rehabilitation against standard therapy in multiple sclerosis patients receiving intravenous steroid treatment. J Neurol Neurosurg Psychiatry 2003;74:1225-30.

8. Francabandera FL, Holland NJ, Wiesel-Levison P, et al. Multiple sclerosis rehabilitation: inpatient vs. outpatient. Rehabil Nurs 1988;13:251-3.

9. Patti F, Ciancio MR, Reggio E, et al. The impact of outpatient rehabilitation on quality of life in multiple sclerosis. J Neurol 2002;249:1027-33.

10. Patti F, Ciancio MR, Cacopardo M, et al. Effects of a short outpatient rehabilitation treatment on disability of multiple sclerosis patients - a randomised controlled trial. J Neurol 2003;250:861-6.

11. Pozzilli C, Brunetti M, Amicosante AMV, et al. Home based management in multiple sclerosis: results of a randomised controlled trial. J Neurol Neurosurg Psychiatry 2002; 73:250-5

12. Stuifbergen $\mathbf{A K}$, Becker $\mathrm{H}$, Blozis $\mathrm{S}$, et al. A randomized clinical trial of a wellness intervention for women with multiple sclerosis. Arch Phys Med Rehabil 2003;84:467-76.

13. Di Fabio RP, Choi T, Soderberg J, et al. Health-related quality of life for patients with progressive multiple sclerosis: influence of rehabilitation. Phys Ther 1997:77:1704-16.

14. Di Fabio RP, Soderberg J, Choi T, et al. Extended outpatient rehabilitation: its influence on symptom frequency, fatigue, and functional status for persons with progressive multiple sclerosis. Arch Phys Med Rehabil 1998;79:141-6. 
15. Storr LK, Sorensen PS, Ravnborg M. The efficacy of multidisciplinary rehabilitation in stable multiple sclerosis patients. Mult Scler 2006;12:235-42.

16. Khan F, McPhail T, Brand C, et al. Multiple sclerosis: Disability profile and quality of life in an Australian Community Cohort. Int J Rehabil Res 2006; 29:87-96.

17. McDonald W, Compston A, Edan G, et al. Recommended diagnostic criteria for multiple sclerosis: guidelines from the International Panel on the Diagnosis of Multiple Sclerosis. Ann Neurol 2001;50:121-7

18. Kurtzke JF. Rating neurological impairment in multiple sclerosis: an expanded disability status scale (EDSS). Neurology 1983;33:1444-52.

19. National Institute for Clinical Excellence. Multiple sclerosis: Management of multiple sclerosis in primary and secondary care. Clinical guidelines, 8 November 2003. National Institute for Clinical Excellence (NHS). ISBN: 1842574426. www.nice. org.uk (accessed 19 July 2008).

20. Granger CV, Cotter AC, Hamilton BB, et al. Functional assessment scales: a study of persons of multiple sclerosis. Arch Phys Med Rehabil 1990;71:870-5.

21. Hobart J, Lamping D, Fitzgerald $\mathrm{R}$, et al. The multiple sclerosis impact scale (MSIS-29): A new patient based outcome measure. Brain 2001; 124:962-73

22. Goldberg DP. Manual of the General Health Questionnaire. Windsor: National Foundation for Educational Research, 1978.

23. Shih WJ. Problems dealing with missing data and informative censoring in clinical trials. Curr Control Trials Cardiovasc Med 2002;3:4.

24. Vickers J, Altman DG. Analysing controlled trials with baseline and follow up measurements. BMJ 2001;323:1123-4.

25. Bland JM, Altman DG. Multiple significance tests: The Bonferroni method. BMJ 1995;310:170

26. Cohen J. Statistical power analysis for the behavioural sciences, 2nd Edn. Hillsdale, NJ: Lawrence Erlbaum Associates, 1988

27. Kazis L, Anderson JJ, Meenan RF. Effect sizes for interpreting changes in health status. Med Care 1989;27:S178-88.
28. Wiles CM, Newcombe RG, Fuller KJ, et al. Controlled randomized cross over trial of the effects of physiotherapy on mobility in chronic multiple sclerosis. J Neurol Neurosurg Psychiatry 2001;70:174-9.

29. Solari A, Filippini G, Gasco P, et al. Physical rehabilitation has a positive effect on disability in multiple sclerosis patients. Neurology 1999;52:57-62.

30. Freeman JA, Langdon DW, Hobart JC, et al. Inpatient rehabilitation in multiple sclerosis: do the benefits carry over into the community? Neurology 1999:52:50-6.

31. Grasso MG, Troisi E, Rizzi F, et al. Prognostic factors in multidisciplinary rehabilitation treatment in multiple sclerosis: an outcomes study. Mult Scler 2005:11:719-24.

32. Langdon DW, Thompson AJ. Multiple sclerosis: a preliminary study of selected variables affecting rehabilitation outcome. Mult Scler 1999;5:94-100.

33. Costello L, O'Rourke K, Kearney H, et al. The patient knows best: significant change in the physical component of the Multiple Sclerosis Impact Scale (MSIS 29). J Neurol Neurosurg Psychiatry 2007;78:841-4.

34. Sprangers MA, Schwartz CE. Integrating response shift into health related quality of life research: a theoretical model. Soc Sci Med 1999;48:1507-15.

35. Benito-Leon J, Morales JM, Riviera-Navarro J. Health quality of life and its relationship to cognitive and emotional function in MS patients. Eur J Neurol 2002;9:497-502.

36. Pfennings $\mathbf{L}$, Cohen $\mathrm{L}$, Ader $\mathrm{H}$, et al. Exploring differences between subgroups of multiple sclerosis patients in health related quality of life. J Neurol 1999:246:587-91.

37. Montel SR, Bungener C. Coping and quality of life in one hundred and thirty five subjects with multiple sclerosis. Mult Scler 2007:13:393-401.

38. Benedict RHB, Carone DA, Bakshi R. Correlating brain atrophy with cognitive dysfunction, mood disturbances and personality disorder in multiple sclerosis. J Neuroimaging 2004:14:36S-45.

39. Khan F, Pallant J. Use of the International Classification of Functioning, Disability and Health (ICF) to identify preliminary comprehensive and brief core sets for multiple sclerosis. Disabil Rehabil 2007;29:205-13.

40. Disler PB, Roy CW, Smith BP. Predicting hours of care needed. Arch Phys Med Rehabil 1993; 78:636-43.

\section{Submit an eletter, and join the debate}

eLetters are a fast and convenient way to register your opinion on topical and contentious medical issues. You can find the "submit a response" link alongside the abstract, full text and PDF versions of all our articles. We aim to publish swiftly, and your comments will be emailed directly to the author of the original article to allow them to respond. eLetters are a great way of participating in important clinical debates, so make sure your voice is heard. 